\title{
The Mental Health Task Force Support Group
}

\author{
John Wattis and Chris Thompson
}

The Mental Health Task Force was set up by the Secretary of State for Health under the leadership of David King to assist in the process of the closure of the large mental hospitals and to ensure adequate provision was made to replace their services. Its strategic objectives, to be accomplished by the end of 1994, were to map the replacement of the remaining large institutions by good quality services, ensuring that this happened effectively; to identify what makes a service good and find ways of ensuring that services possessed these qualities; and to develop a vision of the shape of the mental health market in years to come. To assist in this a wide support group of about 20 people was set up. This included representatives of the Department of Health, Research and Development in Psychiatry, carer organisations, users, social services, general practice, nursing and the Royal College of Psychiatrists. The authors represented the College.

The public output of the task force has been impresstve. Four issues have been produced of a newsletter The Water Tower for managers with a large hospital to replace (a fifth issue is planned). The Water Tower aimed to identify success factors in closing a hospital, report good practice and discuss opportunities and pitfalls. Another newsletter, Grass Roots, more widely circulated to keep people in touch with the work of the task force, has gone to 25 issues. It has focused on good practice as well as highlighting other activities and outputs of the task force. A series of Good Practice videos has been produced on a variety of topics such as 'Alternattves to Hospital' and 'Different Cultures, Different Needs'. These were aimed at purchasers and providers of health care. The support group had no direct involvement with these outputs.

In contrast the support group was deeply involved in a document, Local Systems of Support; a framework for purchasing for people with severe mental health problems, largely written by an American expert, Judy Turner Crowson-but revised by the Department of Health to make sure it fitted political priorities before publication. Here your representatives found themselves fighting hard to ensure recognition of the importance of the 'medical' psychiatric input to mental health and the need for acute hospital provision. In our more paranoid moments it seemed as though there was a conspiracy to de-emphasise the National Health Service's responsibility for people with mental illness although we welcomed the other emphases on housing, leisure opportunities and user and carer involvement. The final document, after intensive lobbying, was a compromise.

The task force was overtaken by the Department of Health's need to react to high profile problems in London's mental health services and Peter Kennedy, a psychiatrist/ general manager from York was brought in to assist in surveying services and preparing a report: Mental Health in London: priorities for action. Interestingly this report was produced with the Department of Health seal rather than under the task force imprintl This important document which will probably be applied in other areas outside London recommended:

(a) increased collaboration between health and local authorities

(b) strengthened mechanisms for communication and co-operation with other key agencies

(c) identifying clearly the population of severely mentally ill people (number. needs and location - the definition of severe mental illness must be agreed with local clinicians)

(d) agreed priorities for development of community based support

(e) making services responsive to people from different ethnic and cultural backgrounds 
(f) addressing pressures on acute psychiatric services with priority to severe mental illness

(g) better purchasing of secure services

(h) no closures of 'long stay institutions' before adequate alternative provision

(i) targeting community mental health team work on people with severe mental illness

(j) developing the care programme approach and supervision registers.

An important issue here is for College members to ensure that local definitions of severe mental Illness include all age groups and categories of disorder that produce severe mental health problems and not only the ones which make front page news.

Further documents on Guidelines for a Local Charter for Users of Mental Health Services, advocacy and mental health services and services for ethnic minority groups are in production. Again the College representatives have had no significant input into these documents. The Guidelines for a Local Charter contain many good ideas about 'user empowerment' but the section on treatment is very negative. Again members will have to be alert that when these guidelines are turned into local 'charters' the negative wording of the section on treatment is modified and that purchasers do not produce charters without regard to the need to fund services to meet the standards set out in those charters.

The task force has sponsored a number of 'stakeholder' and 'user' meetings which the College representatives have only been minimally involved in.

From the College representatives' point of view it has been time-consuming and frustrating trying to ensure that clinical reality has not drowned in newspeak. Some of the task force's public output has been marred by lack of consultation with College representatives but, on the whole, the task force has advanced the cause of services for people with mental illness. Members will need to be vigilant about local definitions of severe mental illness and the 'user's charter' if this advance is to be made real locally.

JoHN WATtis and ChrIS ThOMPSON, College representatives on Mental Health Task Force Support Group

December 1994 